\title{
Hybrid magnetic tunnel junction/ spin filter device
}

\author{
Yuri Suzuki, Brittany Nelson-Cheeseman, Franklin Wong, Rajesh Chopdekar \\ Dept. of Materials Science \& Engineering, UC Berkeley \\ Materials Science Division, LBNL \\ Elke Arenholz \\ Advanced Light Source, LBNL \\ Miaofang Chi, Nigel Browning \\ Dept. of Chemical Engineering and Materials Science, UC Davis \\ Materials Science and Technology Division, LLNL
}

\begin{abstract}
Surfaces and interfaces of complex oxides materials provide a rich playground for the exploration of novel magnetic properties not found in the bulk but also the development of functional interfaces to be incorporated into applications. We have recently been able to demonstrate a new type of hybrid spin filter/ magnetic tunnel junction. Our hybrid spin-filter/magnetic-tunnel junction devices are epitaxial oxide junctions of $\mathrm{La}_{0: 7} \mathrm{Sr}_{0: 3} \mathrm{MnO}_{3}$ and $\mathrm{Fe}_{3} \mathrm{O}_{4}$ electrodes with magnetic $\mathrm{NiMn}_{2} \mathrm{O}_{4}$ barrier layers. Depending on whether the barrier is in a paramagnetic or ferromagnetic state, the junction exhibits magnetic tunnel junction behavior where the spin polarized conduction is dominated by the electrode-barrier interface or spin filter behavior where conduction is dominated by barrier layer magnetism.
\end{abstract}

\section{INTRODUCTION}

The technological potential of spin-based electronics has led many groups to reexamine issues associated with the nature of magnetism at surfaces and interfaces and the manipulation of electron spin. The nature of magnetism at boundaries of spin-polarized materials is a fundamental issue that has yet to be fully understood and may place technological limitations on the implementation and performance of spin polarized devices. In attempts to control or manipulate spin, many researchers have focused on the fabrication and synthesis of magnetic heterostructure devices, including magnetic tunnel junctions (MTJs), spin filters and spin transistors. In a magnetic tunnel junction, two ferromagnetic electrodes sandwich an insulating tunnel barrier. Depending on the spin density of states of the two ferromagnetic electrodes, we observe a high or low resistance. While early theoretical models described the observed junction magnetoresistance in terms of the bulk spin polarization of the electrodes, ${ }^{1}$ recent work has shown the sensitivity of such measurements to interface quality and the spin dependent interface density of states of the electrodes. ${ }^{2}$ Even more recent experimental and theoretical work on magnetic tunnel junctions with $\mathrm{MgO}$ barriers indicate the importance of understanding the role of the barrier layer in the tunneling process, depending on such factors as the chemical bonding between the atoms in the electrodes and atoms in the barrier, interface resonance states, and the symmetries of the propagating states in the electrodes and evanescent states in the barrier. ${ }^{3}$

The introduction of magnetism into the barrier layer provides an additional mechanism through which electron spin can be manipulated. For MTJs with spin-polarized states in the barrier, Jansen et al. found that delta doping of $\mathrm{Al}_{2} \mathrm{O}_{3}$ barriers with $\mathrm{Fe}$ ions increased the junction magnetoresistance (JMR) as a function of Fe content in the barrier. ${ }^{4}$ The enhanced JMR was explained in terms of spin filtering of the tunneling 
electrons, whereby there exists preferential transmission (increased conductance) of one type of spin due to the polarized barrier state. ${ }^{5}$ These results suggest that magnetism in the barrier can directly affect the JMR by being spin preserving rather than adding to spin-flip scattering via spin waves.

Recent studies on MTJs with paramagnetic barriers suggest that the presence of paramagnetism in the barrier layer does not preclude large JMR values or distinct switching characteristics. ${ }^{6-9}$ JMRs as high as $86 \%$ and abrupt switching between parallel and antiparallel configurations have been demonstrated using a paramagnetic $\mathrm{NdGaO} 3$ barrier between two $\mathrm{La}_{0.7} \mathrm{Ca}_{0.3} \mathrm{MnO}_{3}$ electrodes. ${ }^{6} \mathrm{JMRs}$ of up to $-25 \%$ were reported in MTJs of $\mathrm{Fe}_{3} \mathrm{O}_{4} / \mathrm{CoCr}_{2} \mathrm{O}_{4} / \mathrm{La}_{0.7} \mathrm{Sr}_{0.3} \mathrm{MnO}_{3}$, where $\mathrm{CoCr}_{2} \mathrm{O}_{4}$ is a material with $\mathrm{T}_{C}$ of $95 \mathrm{~K}$ in the bulk form. ${ }^{7}$

We have developed a hybrid magnetic tunnel junction/ spin filter device in which spin transport is tuned via the magnetic state of the barrier layer. ${ }^{10-11}$ In particular, we have studied $\mathrm{Fe}_{3} \mathrm{O}_{4} / \mathrm{NiMn}_{2} \mathrm{O}_{4}(\mathrm{NMO}) / \mathrm{LSMO}$ (spinel/spinel/perovskite) junctions. Spin polarized transport measurements reveal junction magnetoresistance values as high as $-30 \%$ (normalized to $8 \mathrm{kOe}$ ) and three transport regimes that reveal two different conduction mechanisms. Above the $\mathrm{T}_{C}$ of the NMO barrier, when the barrier layer is paramagnetic, the electrode-barrier interfaces dominate the spin transport, resulting in an asymmetric bias dependence of the JMR and inelastic tunneling spectra (IETS), characteristic of the dissimilar electrode-barrier interfaces. Below the $\mathrm{T}_{C}$ of the NMO barrier, the ferrimagnetism in the barrier begins to dominate the spin transport, resulting in a transition to a symmetric bias dependence of the JMR and IETS. X-ray magnetic circular dichroism (XMCD) confirms the $\mathrm{T}_{C}$ of the $\mathrm{NMO}$ barrier to be $60 \mathrm{~K}$, as well as the nature of the magnetic coupling at the electrode/barrier interfaces.

\section{EXPERIMENTAL METHODS}

Trilayer heterostructures of $\mathrm{Fe}_{3} \mathrm{O}_{4} / \mathrm{NMO} / \mathrm{LSMO}$ were grown by pulsed laser deposition (PLD) on (110) $\mathrm{SrTiO}_{3}$ substrates. PLD was performed with a $\mathrm{KrF}$ excimer laser $(248 \mathrm{~nm})$ at $10 \mathrm{~Hz}$ with energy density of $\sim 2 \mathrm{~J} / \mathrm{cm}^{2}$. The LSMO layer was grown in $320 \mathrm{mTorr}$ of $\mathrm{O}_{2}$ at $700{ }^{\circ} \mathrm{C}$; the insulating NMO layer was grown in 10 mTorr of $99 \% \mathrm{~N}_{2} / 1 \% \mathrm{O}_{2}$ gaseous mixture at $550{ }^{\circ} \mathrm{C}$; and the $\mathrm{Fe}_{3} \mathrm{O}_{4}$ layer was grown in vacuum $\left(\sim 4 \times 10^{-6}\right.$ Torr $)$ at $400{ }^{\circ} \mathrm{C}$. The LSMO and $\mathrm{Fe}_{3} \mathrm{O}_{4}$ layers are typically 600-800 $\AA$ thick, with the NMO barrier layer thickness ranging from 20 45 $\AA$. Both LSMO and $\mathrm{Fe}_{3} \mathrm{O}_{4}$ are thought to be half metallic theoretically and have been shown experimentally to be highly spin polarized, thus making them ideal candidates for magnetic junction electrodes. ${ }^{12-14}$

X-ray diffraction was used to verify epitaxy as well as crystalline quality of the heterostructures. Rutherford backscattering spectroscopy was also used to determine the thickness and stoichiometry of our samples. High-resolution transmission electron microscopy (TEM) was performed on a Philips CM300 in order to examine the structure of the film-substrate interface.

The bulk magnetic properties of the films were measured with a superconducting quantum interference device magnetometer. Interface magnetism was investigated by X-ray magnetic circular dichroism (XMCD) at $30^{\circ}$ grazing incidence for the $\mathrm{Mn}$ and $\mathrm{Fe}_{2,3}$ edges at the Advanced Light Source on beamline 4.0.2. XMCD provides element specific surface sensitive magnetic information through the subtraction of X-ray absorption spectroscopy data for right and left circularly polarized light. Photoemission electron microscopy at the Advanced Light Source on beamline 11.0.1 has provided imaging of magnetic contrast in our trilayers.

The films were fabricated into junctions by contact alignment photolithography and Ar ion milling into squares of area $4 \times 4,6 \times 6,10 \times 10,20 \times 20$, and $40 \times 40 \mu \mathrm{m}^{2} . \mathrm{SiO}_{2}$ was deposited to isolate the top and bottom contact pads. Magnetotransport measurements of the junctions were taken in the current perpendicular to the plane geometry, with the applied magnetic field oriented along the in-plane (001) direction, which is the magnetically easy direction for both LSMO and $\mathrm{Fe}_{3} \mathrm{O}_{4}$ films grown on (110) STO. The JMR was 
calculated in accordance with Julliere's model by the following equation: $\left(\Delta R / R_{P}\right) * 100$ where $\Delta \mathrm{R}=\mathrm{R}_{\mathrm{AP}}-\mathrm{R}_{\mathrm{P}}$. The reference (parallel magnetization) resistance was taken as the resistance at $8 \mathrm{kOe}$.

\section{RESULTS}

\subsection{Structure}

X-ray diffraction confirmed that the heterostructures were epitaxial with (110) orientation of the electrodes and barrier layer out-of-plane as well as in-plane alignment of the crystallographic directions of the layers. Figure 1 shows a typical X-ray diffraction pattern of a trilayer sample. LSMO has a cubic perovskite structure with a bulk lattice parameter of 3.87A. NMO and $\mathrm{Fe}_{3} \mathrm{O}_{4}$ both crystallize in the spinel structure with lattice parameter of 8.39 and $8.394 \AA$ respectively. Despite the relatively large lattice mismatch between the perovskite and spinel films, the spinel layers grow coherently on the LSMO with crystalline registry and good crystalline quality. Figure 2 shows a typical TEM micrograph of the interface in cross sectional view. We observe flat and coherent perovskite/ spinel interfaces. However, it is difficult to discern the spinel/ spinel interface due to the similar $\mathrm{Z}$ values of the cations. However, even with the relatively high crystalline quality of the spinel films, the large lattice mismatch between the perovskite and spinel structures inevitable creates defects at the perovskite-spinel interface as well as the spinel layers themselves. Although the spinel films are not perfectly epitaxial, a combination of low-angle grain boundaries, anti-phase boundaries, and dislocations act as mechanisms for lattice relaxation that allow the spinel films to maintain good structural registry with the perovskite underlayer.

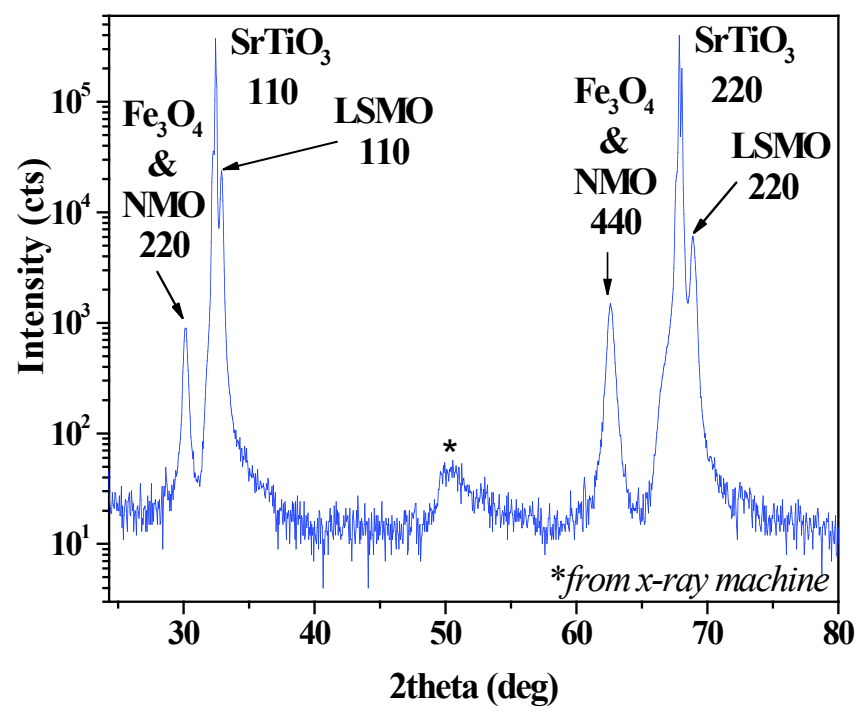

Figure 1. XRD of a typical layer shows (110) orientation of both the perovskite and spinel layers.

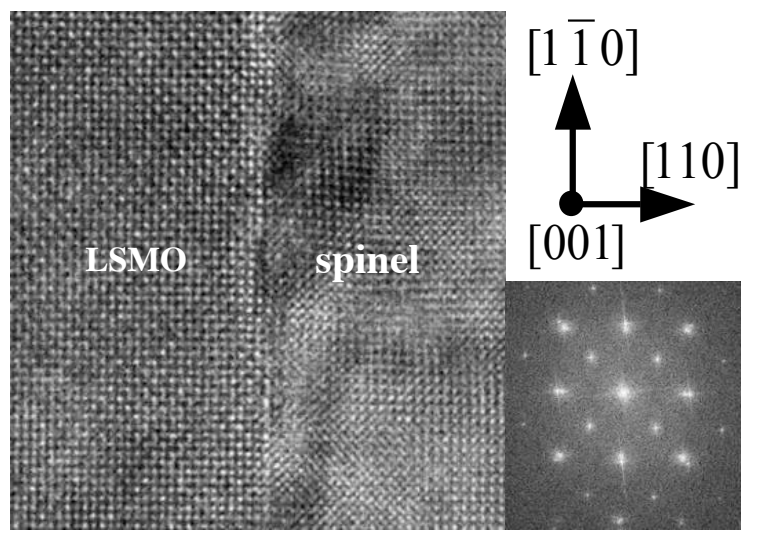

Figure 2. $\mathrm{LSMO} / \mathrm{NMO} / \mathrm{Fe}_{3} \mathrm{O}_{4}$ trilayer. (a) Cross sectional TEM and (b) electron diffraction, demonstrating good crystallinity and texture of both the perovskite and spinel as well as abrupt interface termination.

\subsection{Magnetism}

Magnetic hysteresis loops of the trilayers at $300 \mathrm{~K}$ show distinct switching of the two ferromagnetic layers with coercive fields of $100 \mathrm{Oe}$ and $700 \mathrm{Oe}$ corresponding to the LSMO and $\mathrm{Fe}_{3} \mathrm{O}_{4}$ ferromagnetic layers, respectively. This large coercive difference and the sharpness of the switching create well-defined parallel and antiparallel magnetization states. 


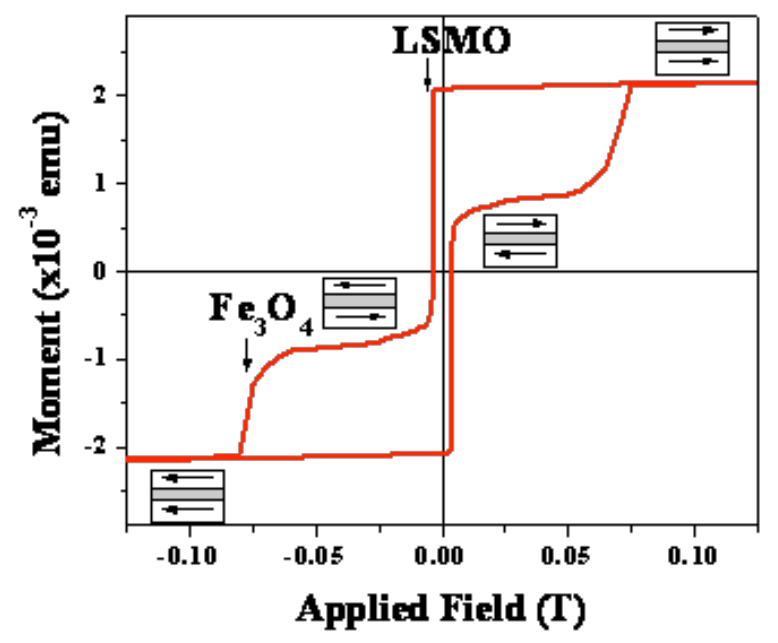

Figure 3. Hysteresis loop of a typical trilayer showing the distinct switching of the softer LSMO and harder $\mathrm{Fe}_{3} \mathrm{O}_{4}$ layer.

antiparallel magnetization states. The absence of strong exchange coupling between the electrodes allows for free switching of the magnetization in the electrodes. As shown in Figure 3, transitions in the magnetization hysteresis loops coincide well with large and abrupt transitions in the junction magnetoresistance.

Since the NMO barrier layer is only $20-45 \AA$ thick, it is difficult to probe the magnetic properties of the barrier layer with bulk techniques such as SQUID magnetometry. Therefore, we have used XMCD of trilayer heterostructures as well as of truncated heterostructures, composed of the NMO barrier layer and bottom LSMO electrode. These two types of samples enable us to probe the magnetism at the isostructural spinel interface as well as the spinel-perovskite interface. Figure 4 shows the $\mathrm{Mn} \mathrm{L}_{2,3}$ XMCD spectra as well as hysteresis loops performed at two different incident X-ray energies. There is a large peak $(640 \mathrm{eV})$ and valley $(642.5 \mathrm{eV})$ observed in the XMCD spectra at low temperature that is reminiscent of spectra from a thick NMO thin film. ${ }^{15}$ This feature is not observed in Mn XMCD spectra at 80 and $100 \mathrm{~K}$ which resemble spectra more like that of LSMO thin films. ${ }^{16}$ In order to determine which features are associated with the Mn in the NMO and in the LSMO respectively, hysteresis loops were taken at 640 and $642.5 \mathrm{eV}$. Hysteresis loops at $642.5 \mathrm{eV}$ show magnetically soft $\mathrm{Mn}$ with coercive fields $\sim 0.5 \mathrm{kOe}$ over the entire temperature range. Those taken at $640 \mathrm{eV}$ show soft magnetic loops above $60 \mathrm{~K}$, similar to those at $642.5 \mathrm{eV}$, while those at lower temperatures are magnetically harder and do not reach saturation by $8 \mathrm{kOe}$. These hard loops resemble SQUID hysteresis loops of single NMO films. This transition indicates the onset of ferrimagnetism in the magnetically hard NMO barrier is at $60 \mathrm{~K}$.

From the XMCD data, it is clear that at $640 \mathrm{eV}$ the Mn magnetism in both the NMO and LSMO are probed whereas only the LSMO magnetism is probed at $642.5 \mathrm{eV}$. A close look at the hysteresis loops at $55 \mathrm{~K}$ taken at $640 \mathrm{eV}$ and $642.5 \mathrm{eV}$ provides strong evidence that the NMO and LSMO layers are not magnetically coupled. The lack of magnetic coupling at the LSMO/NMO interface likely arises from the presence of misfit dislocations at the LSMO/NMO interface due to the lattice mismatch between the perovskite and spinel structures. Such dislocations would provide for easy $180^{\circ}$ domain wall creation at the LSMO/NMO interface. 


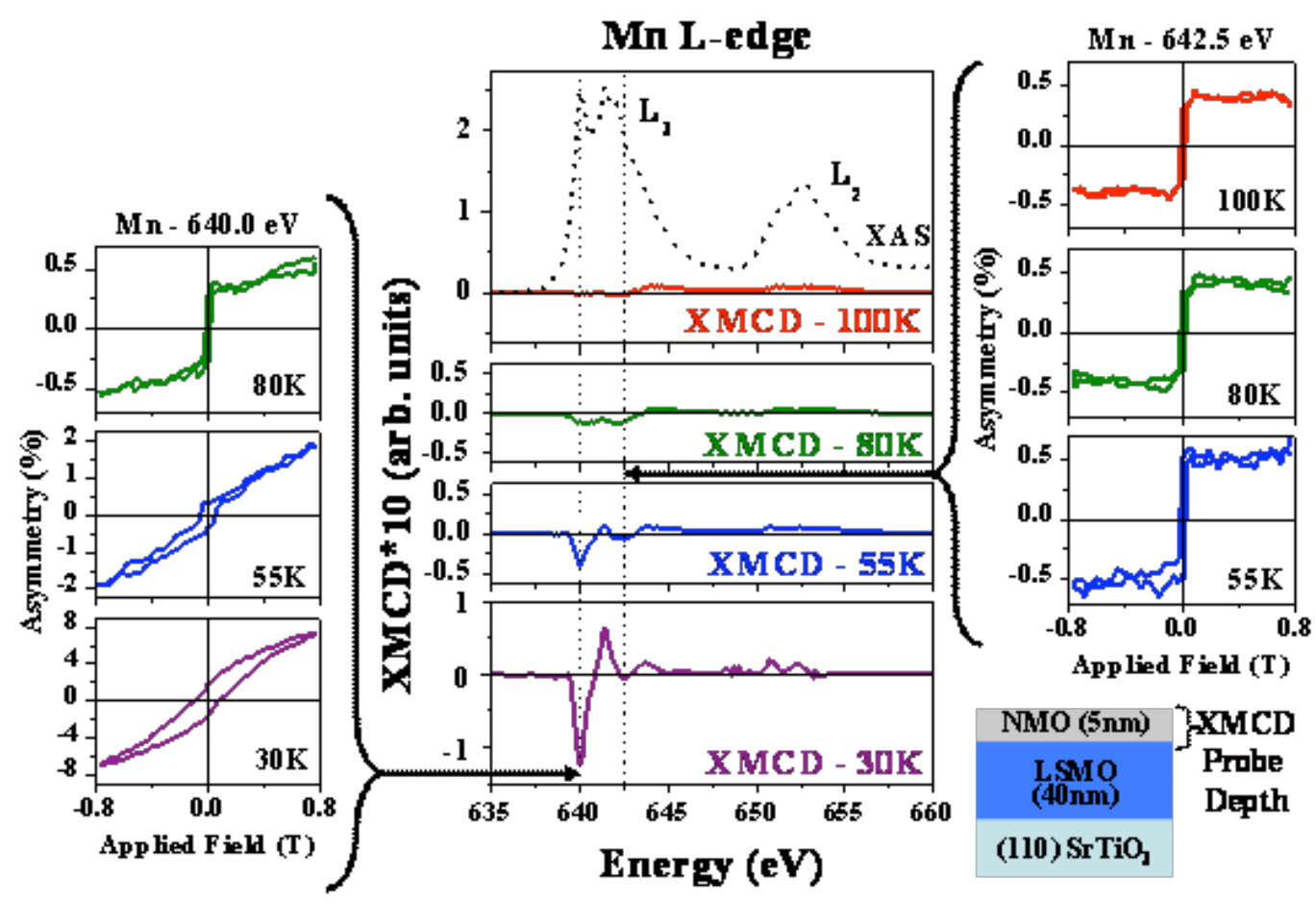

Figure 4. X-ray magnetic circular dichroism was used to investigate the magnetism at the LSMO/NMO interface. Mn XMCD spectra of NMO/LSMO interface, and Mn hysteresis loops taken at $640.0 \mathrm{eV}$ and $642.5 \mathrm{eV}$ are shown as a function of temperature.
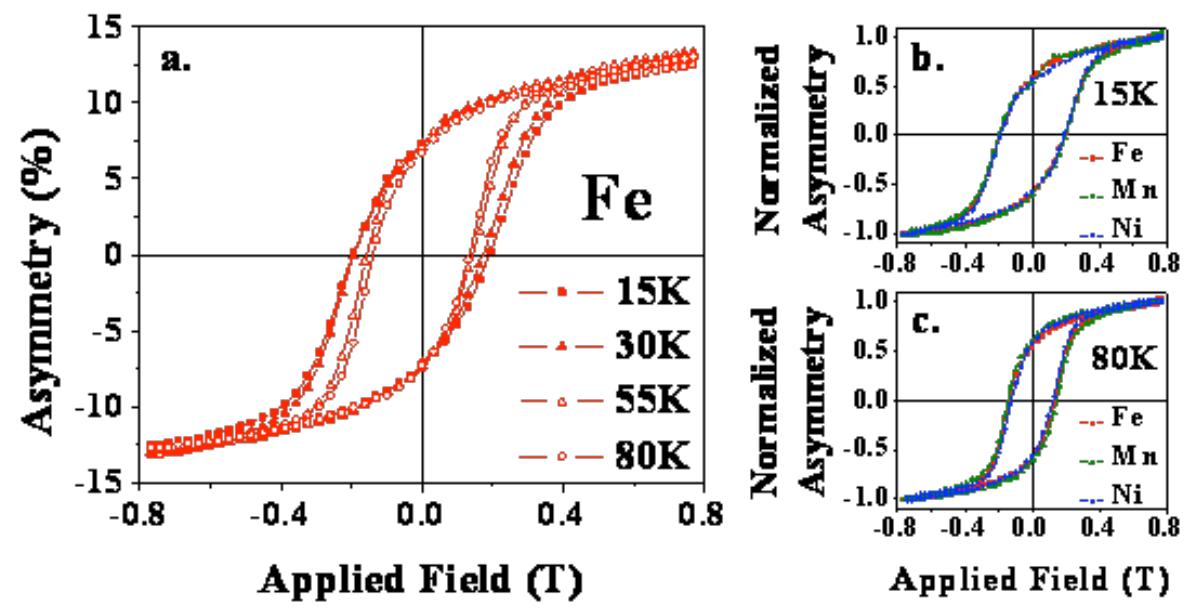

Figure 5. X-ray magnetic circular dichroism Fe hysteresis loops as a function of temperature, taken probing the top 5-6nm of the trilayer sample shown. Figures 5(b) and (c) show coincident Fe, Mn and Ni XMCD loops at 15 and 80K. 
It is the lack of coupling at this interface that gives rise to the independent switching of the two electrodes below the magnetic ordering temperature of the NMO. The independent switching is further verified in symmetric minor loops of LSMO.

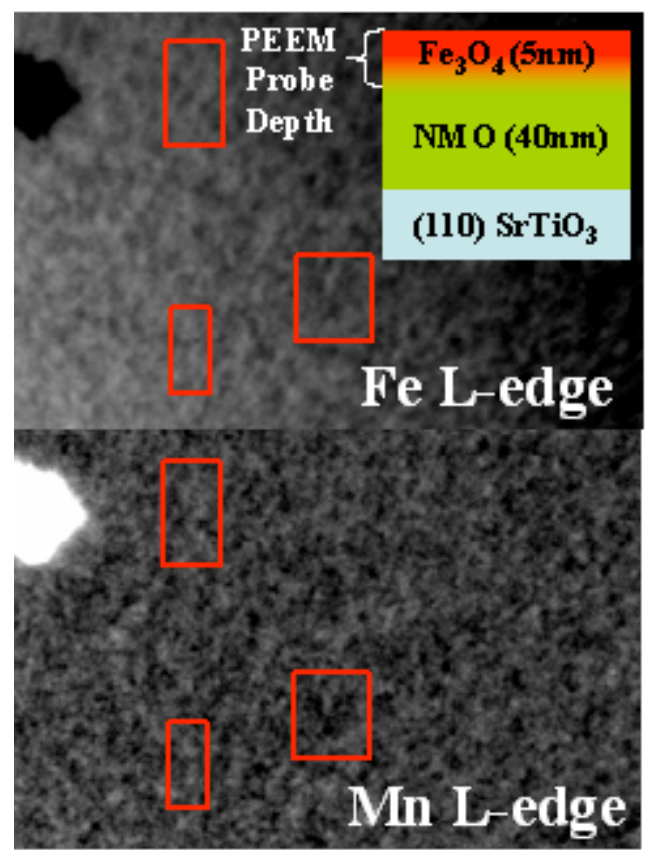

Figure 6. PEEM image of the Fe and Mn edges indicate that the magnetic species are coupled antiferromagnetically. The boxed areas show coincident magnetic contrast.
The $\mathrm{NMO} / \mathrm{Fe}_{3} \mathrm{O}_{4}$ interface exhibits $\mathrm{Fe}, \mathrm{Ni}$, and $\mathrm{Mn}$ dichroism up to room temperature, as well as a direct coupling of the $\mathrm{Ni}, \mathrm{Mn}$, and Fe XMCD hysteresis loops at all temperatures (Fig. 5). The coincidence of the $\mathrm{Ni}, \mathrm{Mn}$ and $\mathrm{Fe}$ hysteresis loops at $15 \mathrm{~K}$ and $80 \mathrm{~K}$ (Figure 5 (b) and (c)) indicate that the three magnetic species are strongly coupled to one another. Although the $\mathrm{Ni}$, $\mathrm{Mn}$ and Fe XMCD hysteresis loops from the trilayer sample are concurrent for all temperatures, the shape of the hysteresis loops changes distinctly below $55 \mathrm{~K}$, exhibiting magnetically harder hysteresis loops. This change indicates that above 55K (NMO $\mathrm{T}_{\mathrm{c}}$ ) the hysteresis loop is dominated by $\mathrm{Fe}_{3} \mathrm{O}_{4}$ whereas below $55 \mathrm{~K}$ the hysteresis loop is dominated by the magnetically hard NMO exchange coupled to the $\mathrm{Fe}_{3} \mathrm{O}_{4}$. In addition, while the $\mathrm{Ni}$ signal was too low to resolve, room temperature PEEM images of $\mathrm{NMO}(40 \mathrm{~nm}) / \quad \mathrm{Fe}_{3} \mathrm{O}_{4} \quad(5 \mathrm{~nm})$ bilayers show antiferromagnetically coincident $\mathrm{Fe}$ and $\mathrm{Mn}$ magnetic domains (Figure 6).

\subsection{Transport}

Transport measurements on the trilayer junctions included junction magnetoresistance as a function of applied field, temperature and bias as well as bias dependence of the conductivity and inelastic tunneling spectroscopy.

The transport of the fabricated junctions exhibit square junction magnetoresistance (JMR) loops with a level background magnetoresistance (MR) at high magnetic fields. As shown in Figure 7, jumps in the magnetic hysteresis loops correlate with large and abrupt jumps in the JMR. When the two magnetic electrodes are magnetized antiparallel to one another, there arises a low resistance state and thus negative JMR values. This negative JMR is due to the opposite spin polarizations of the LSMO and $\mathrm{Fe}_{3} \mathrm{O}_{4}$ electrodes, which are majority and minority spin polarized, respectively. ${ }^{7}$ The background magnetoresistance (MR) as a fraction of the maximum JMR for these junctions, as shown in Figure 7, is significantly lower than that previously seen in similar LSMO-Fe $\mathrm{O}_{4}$ junctions with other spinel barrier layers. ${ }^{7-8}$

The bias dependence of the conductivity was also studied to determine the transport mechanisms active in the junctions. The IV curves were highly nonlinear at all temperatures, indicating that the NMO is an effective barrier both above and below its Curie temperature. The bias dependence of the conductance (dI/dV) reveals the dominant conduction mechanisms at different voltage levels. ${ }^{17}$ At all temperatures, a single fit of the entire voltage range $(0$ to $100 \mathrm{mV})$ did not accurately describe the data. However, separate fitting of the low bias and high bias regions yielded excellent data fits shown in Figure 8, indicating that there are distinct conduction mechanisms at low and high bias. At low bias the conductance is best fit by a $\mathrm{V}^{2}$ dependence indicative of elastic tunneling, while at high bias the conductance is best fit by a $\mathrm{V}^{4 / 3}$ dependence indicative of inelastic hopping transport through up to two barrier sites. The boundary between the two conduction regimes 


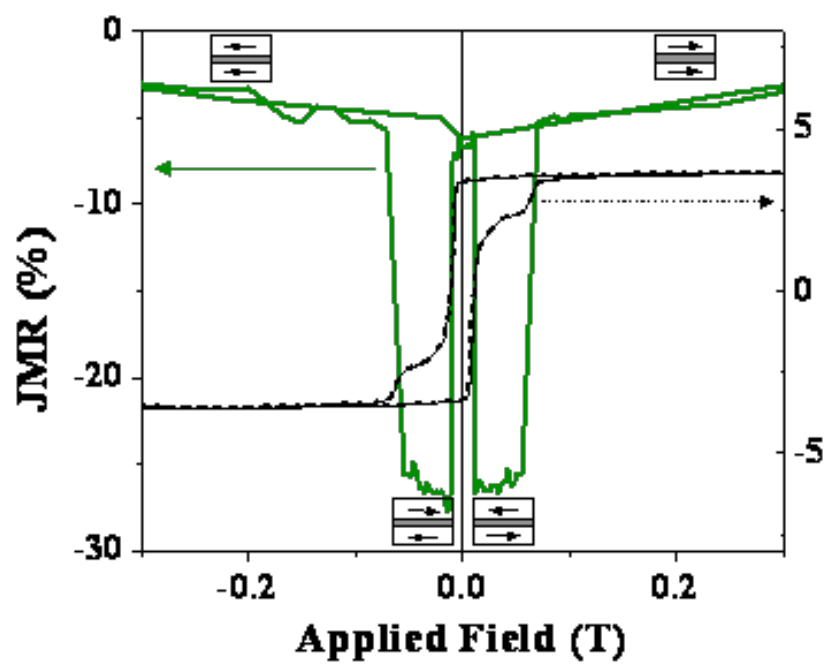

Figure 7. Junction transport as a function of applied magnetic field at $75 \mathrm{~K}$. (a) JMR and moment at low magnetic fields, (b) JMR at high magnetic fields.

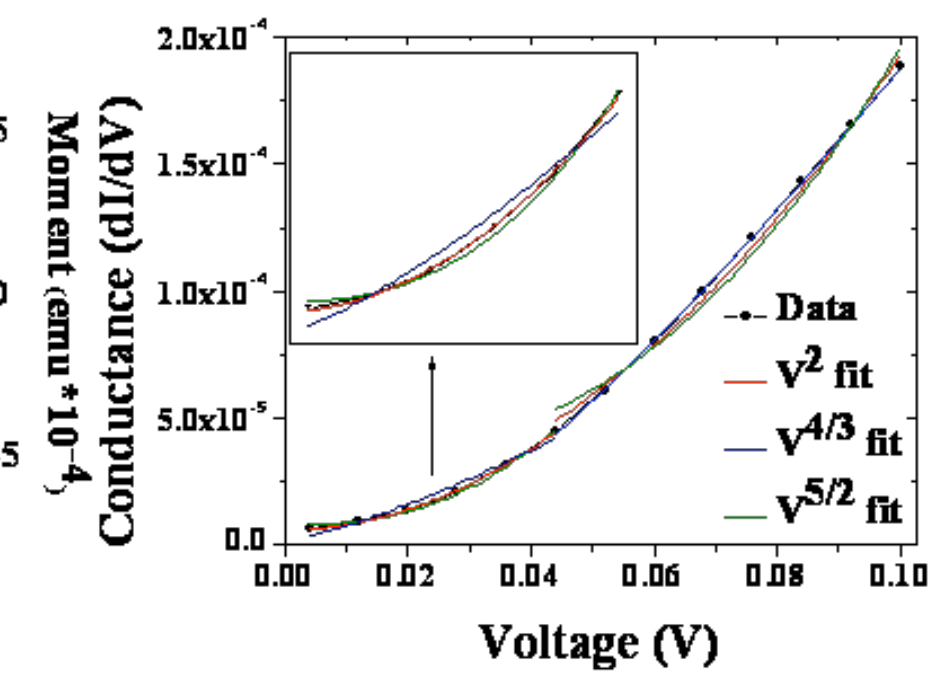

Figure 8. Low bias data of the conductance show $\mathrm{V}^{2}$ dependence indicative of elastic tunneling across barrier, while high bias data show $\mathrm{V}^{4 / 3}$ dependence indicative of inelastic hopping through two sites.

occurs between $40-60 \mathrm{eV}$, and coincides with a change in curvature of the conductance curve. The inelastic hopping that occurs at higher bias likely results from phonons, magnons at the electrode-barrier interfaces, magnons within the barrier layer when it is ferrimagnetic, as well as defects within the barrier layer.

Plots of JMR as a function of temperature and applied voltage reveal three transport regimes with two different conduction mechanisms. Figure 9 shows JMR as a function of temperature for a typical trilayer junction where there is a distinct maximum in the absolute value of JMR at low temperatures. This maximum is surprising since the spin polarization of the electrodes should increase as a function of decreasing temperature. However, similar behavior has been observed in LSMO/spinel/ $\mathrm{Fe}_{3} \mathrm{O}_{4}$ junctions with spinel barriers of $\mathrm{CoCr}_{2} \mathrm{O}_{4}, \mathrm{MgTi}_{2} \mathrm{O}_{4}$ and $\mathrm{FeGa}_{2} \mathrm{O}_{4}{ }^{7-8}$ and has been attributed to the increase in the $\mathrm{Fe}_{3} \mathrm{O}_{4}$ electrode resistance that occurs near its Verwey transition (metal-insulator) transition. A closer look at the bias dependence of the JMR reveals that there are in fact three transport regimes. At high temperatures, the bias dependence drops off gradually at higher voltages and is asymmetric with the JMR maximum at finite voltage. A transition occurs around $60 \mathrm{~K}$, where the bias dependence transitions to a symmetric one, characterized by a JMR maximum at zero bias, a highly symmetric shape, and a rapid decrease in JMR at higher bias. At low temperatures, the bias dependence continued to be symmetric about zero bias, with decreasing JMR with decreasing temperature attributed to the increasing electrode resistance relative to the junction resistance.

In order to explain the transition from asymmetric to symmetric bias dependence in the JMR, inelastic transport spectroscopy (IETS) was performed. Basically, this is a plot of $\mathrm{d}^{2} \mathrm{I} / \mathrm{d}^{2} \mathrm{~V}$ as a function of voltage and probes the inelastic tunneling processes due to phonons and magnons in the material. In order to probe just the magnon effects, we have subtracted the IETS spectra of the antiparallel magnetization configuration from that of the parallel magnetization configuration and plotted the difference in Figure 10 . The $d^{2} I / d^{2} V=0$ value occurs at a finite bias for $T \sim 60 \mathrm{~K}$, but moves towards zero bias below $60 \mathrm{~K}$. The peak locations (30-50 $\mathrm{mV}$ ) of the IETS of our junctions for all temperatures are well within the range of 12-100 $\mathrm{mV}$ 
commonly associated with magnons. ${ }^{18-19}$ The observation of symmetric at lower temperatures and asymmetric bias dependence at higher temperatures in the same junction is surprising and suggests that at higher temperatures magnons associated with the interface dominate the transport whereas at lower temperatures magnons associated with the NMO barrier layer itself dominate the transport.

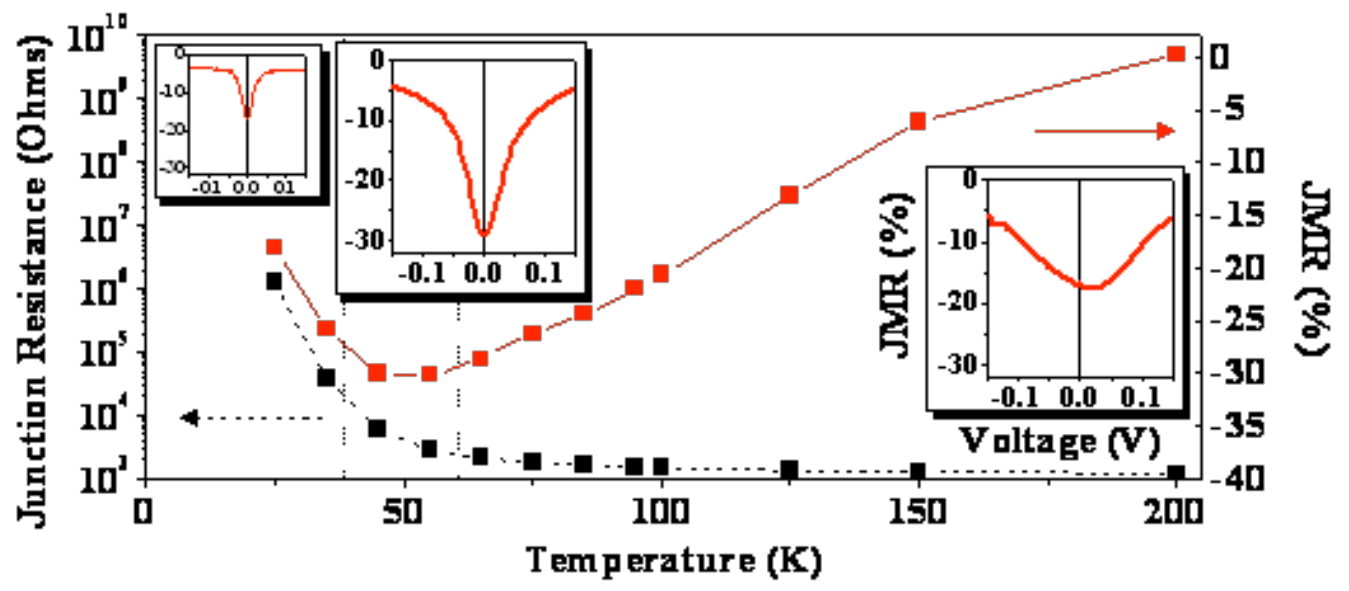

Figure 9. JMR as a function of temperature and bias (insets) indicate three different transport regimes.

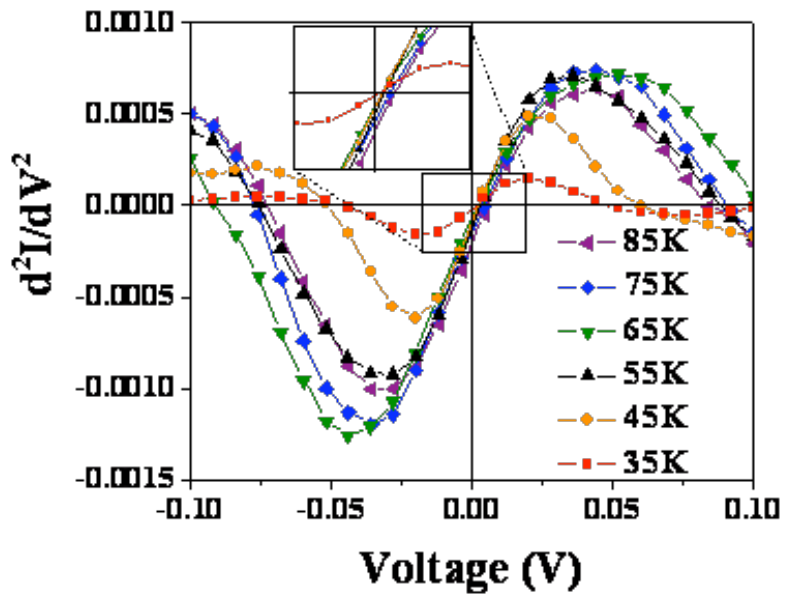

Figure 10. IETS as a function of temperature, showing shift towards symmetry about zero bias below $65 \mathrm{~K}$.

\section{CONCLUSIONS}

In conclusion, we have demonstrated a heterostructure in which spin filtering behavior is observed at low temperatures and magnetic tunneling behavior is observed at higher temperatures. Spin transport is controlled by interfaces in the magnetic tunneling regime or by the insulating barrier in the spin filtering regime. This is seen by a distinct change in the bias dependence of the JMR that coincides with the onset of ferrimagnetism in the NMO barrier layer as confirmed by XMCD. When the barrier layer is paramagnetic at high temperatures, the electrode-barrier interfaces characterize the asymmetric JMR bias dependence due to their disparate electrode-barrier heights and interface magnon properties. As the temperature is lowered, the JMR 
bias dependence transitions to a highly symmetric nature suggesting a conduction mechanism inherent to the bulk barrier, specifically spin flip scattering caused by bulk-like magnons within the ferrimagnetic NMO barrier layer. These magnetic heterostructures are the first manifestation of a hybrid magnetic tunnel junction/ spin filter device.

\section{ACKNOWLEDGEMENTS}

This work was supported in full by the Office of Basic Energy Sciences, Division of Materials Sciences and Engineering, of the U.S. Department of Energy under Contract No. DE-AC02-05CH11231. Thanks to J. S. Moodera for fruitful discussion. Processing was performed in the University of California Berkeley Microlab. The Advanced Light Source is supported by the Director, Office of Science, Office of Basic Energy Sciences, of the U.S. Department of Energy under Contract No. DE-AC02-05CH11231.

\section{REFERENCES}

[1] Julliere, M., Phys. Lett. 54A, 225 (1975).

[2] Woods, G.T., Soulen, Jr., R.J., Mazin, I., Nadgorny, B., Osofsky, M.S., Sanders, J., Srikanth, H., Egelhoff and Datla, R., Phys. Rev. B. 70, 054416 (2004).

[3] Butler, W.H., Zhang, X.-G., Schulthess, T.C., and MacLaren, J.M., Phys. Rev. B 63, 054416 (2001).

[4] Jansen, R. and Moodera, J.S. Appl. Phys. Lett. 75, 400 (1999).

[5] Jansen, R. and Lodder, J. C., Phys. Rev. B 61, 5360 (2000).

[6] Jo, M-H., Mathur, N.D., Todd, N.K., and Blamire, M.G., Phys. Rev. B 61, 14905 (2000).

[7] Hu, G. and Suzuki,Y. Phys. Rev. Lett. 89, 276601 (2002).

[8] Alldredge, L.M.B., Chopdekar, R.V.,. Nelson-Cheeseman, B.B and Suzuki,Y., J. Appl. Phys. 99, 08K303 (2006).

[9] Alldredge, L.M.B., Chopdekar, R.V.,. Nelson-Cheeseman, B.B and Suzuki,Y.,, Appl. Phys. Lett. 89, 182504 (2006).

[10] Nelson-Cheeseman, B.B., Chopdekar, R.V., Alldredge, L.M.B., Bettinger, J.S., Arenholz, E., Suzuki, Y., Phys. Rev. B. 76, R220410 (2007)

[11] Nelson-Cheeseman, B.B., Chopdekar, R.V., Bettinger, J.S., Pickett, M., Arenholz, E., Marcus, M., Suzuki, Y., J. Appl. Phys. 103, 07B524 (2008).

[12] Picket, W.E. and. Singh, D.J, J. Magn. Magn. Mater. 172, 237 (1997).

[13] Yanase, A. and Siratori, K., J. Phys. Soc. Jpn. 53, 312 (1984).

[14] Zhang, Z. and Satpataphy,S., Phys. Rev. B 44, 13319 (1991).

[15] Nelson-Cheeseman, B.B., Chopdekar, R.V., Toney, M., Bettinger, J.S., Arenholz, E., Suzuki, Y., unpublished.

[16] Stadler, S., Idzerda, Y. U., Chen, Z., Ogale, S.B., and Venkatesan, T., Appl. Phys. Lett. 75, 3384 (1999).

[17] Glazman, L.I. and Matveev, K.A., Sov. Phys. JETP 67, 1276 (1988).

[18] Tsui, D.C., Dietz, R.E, Walker, L.R., Phys. Rev. Lett. 27, 1729 (1971).

[19] Ando, Y., Murai, J., Kubota, H., and Miyazaki, T., J. Appl. Phys. 87, 5209 (2000). 